\title{
LA EDUCACIÓN TÉCNICO PROFESIONAL Y LAS COMPETENCIAS PARA LA CIUDADANÍA. EL CASO DE LAS COMUNAS DE LA PROVINCIA DE CONCEPCIÓN, CHILE ${ }^{1}$
}

\author{
Olga Carrillo \\ Pedro Jurado ${ }^{3}$
}

\begin{abstract}
RESUMEN
El estudio aborda el tema de las competencias para la ciudadanía en la enseñanza media técnico profesional en las comunas de la provincia de Concepción en Chile. Sus objetivos son analizar el nivel de logro alcanzado respecto de las competencias para la ciudadanía por los alumnos/as de esta modalidad educativa, así como también estudiar la importancia percibida por parte de ellos hacia dichas competencias. Se utilizó un diseño secuencial explicativo, la muestra fue de tipo no probabilístico $(n=422)$ y se establecieron criterios para la selección de los informantes para el apartado cualitativo ( $n=34,17$ profesores y 17 estudiantes). Los instrumentos utilizados para la recolección de la información fueron el cuestionario de autopercepción de competencias ciudadanas y la entrevista semiestructurada. Los resultados de esta investigación muestran que los estudiantes tienen una alta autopercepción de las competencias ciudadanas $(M=3,34)$, lo que se complementa con el análisis de las entrevistas, donde queda de manifiesto que el logro de las competencias se vincula, por un lado, con el desarrollo del currículo en esta modalidad educativa -que incluye prácticas en las empresas-y, por otro, con la conducta y características personales de los/las estudiantes.
\end{abstract}

Palabras clave: ciudadanía, competencias, educación vocacional, estudiantes, formación profesional.

\section{TECHNICAL-PROFESSIONAL EDUCATION AND CITIZENSHIP COMPETENCIES. THE CASE OF THE MUNICIPALITIES OF CONCEPCIÓN PROVINCE, CHILE.}

\section{ABSTRACT}

This study addresses the theme of civic competencies in technical professional secondary education in the municipalities of Concepción Province, Chile. The objectives are focused on analyzing the level of achievement with regards to the students' civic competencies in this educational modality, as well as analyzing the student's perceived importance of said competencies. The sequential explanatory model was used. The sample was non-probabilistic $(n=422)$ and, in the qualitative portion, a selection

Este trabajo fue financiado por el Programa de Capital Humano Avanzado Conicyt - Becas Chile.

2 Facultad de Educación, Universidad Católica de Temuco, Temuco, Chile. Contacto: ocarrillo@uct.cl

3 Facultad de Ciencias de la Educación, Universidad Autónoma de Barcelona, Barcelona, España. Contacto: pedro.jurado@uab.cat 
criteria for the informants was established ( $n=34: 17$ teachers and 17 students). The instruments used for the data collection were a questionnaire about self-perception of civic competencies and a semi-structured interview. The results of this investigation demonstrate that the students possess a high self-perception of their civic competencies $(M=3.34)$. This is complemented by the analysis of the interviews, where it is evidenced that the acquisition of these competencies is linked, on the one hand, to the development of the curriculum in this educational modality, which includes company internships, and, on the other hand, by the students' conduct and characteristics.

Keywords: civic education, competencies, professional training, students, vocational education.

\section{Introducción}

La formación para la ciudadanía es un pilar fundamental para el fortalecimiento y sustento de una sociedad en la que predominan los valores democráticos, donde el respeto, la igualdad y la responsabilidad son los elementos que garantizan la convivencia social en un entorno de diversidad. Para ello es necesario que los sujetos adquieran los aprendizajes de lo que se supone es este tipo de relación social y, en ese sentido, la escuela constituye la primera experiencia (Bolívar, 2007; Gimeno, 2002; Peña, 2015).

Ser ciudadano es un símbolo de igualdad a la que aspiran las sociedades democráticas -sociedades diversas, pluriculturales- en las que conviven y se interrelacionan hombres y mujeres que reconocen para sí una misma pertenencia, la que además de generar vínculos y límites con el Estado, establece responsabilidades para el sustento del sistema democrático (Ley Orgánica Constitucional de Enseñanza, LOCE, 1990; Ley General de Educación, LGE, 2009).

La formación ciudadana es importante para garantizar la permanencia de los valores democráticos indispensables para la construcción de sociedades dinámicas y justas. Por ello, es fundamental que este tipo de formación sea considerada como una herramienta necesaria para que los niños, niñas y jóvenes se conviertan en ciudadanos activos y responsables. Desde esta perspectiva, la ciudadanía no se debe limitar solamente al estatus jurídico y político -tras lo cual se adquieren derechos y responsabilidades-, sino que se debe visualizar ampliamente, promoviendo la participación en los asuntos públicos, ya sean estos locales, nacionales o globales; y concibiéndola desde diversos escenarios: ambientales, sociales y 
multiculturales (Banks, 2008; Banks et al., 2005; Maiztegui, 2007; Pagés y Santiesteban, 2009; Tyson y Choon, 2008). De este modo, la ciudadanía viene siendo el acto de ejercer derecho a elección, a la libertad de expresión, a la participación en voluntariados, a la vez que implica la capacidad de definir cuáles son los problemas, cómo serán abordados y solucionados (Magendzo, 2003).

Las necesidades actuales impulsan la inclusión de nuevos aspectos en la educación como los psicológicos, sociales, políticos y culturales, por lo que en este escenario esta posee un rol fundamental al potenciar habilidades y capacidades para llevar a cabo el ejercicio ciudadano. La escuela debe dotar de experiencias de vida democrática y de ciudadanía "que trasciendan las formas de vida en competencia o la particularidad de la familia” (Peña, 2015, p. 35).

En este sentido, en Chile se han realizado estudios que han abordado la temática a partir de los derechos humanos en el currículo de Historia y Ciencias Sociales de $2^{\circ}$ año medio (Magendzo y Toledo, 2009); la formación ciudadana desde el ejercicio docente y cómo esta se afronta en el aula (Reyes, Campos, Osandón y Muñoz, 2013); la formación ciudadana a partir de las percepciones de los estudiantes (Muñoz y Torres, 2014; Muñoz, Vásquez y Reyes, 2010); así como también las prácticas docentes, representaciones y actitudes ciudadanas de jóvenes desde la perspectiva de los propios actores involucrados (Bonhomme, Cox, Tham y Lira, 2015). A la vez, se han realizado estudios internacionales como el de educación cívica y formación ciudadana ( $\mathrm{ICCS}^{4}$ ) que ha contribuido al tema desde una perspectiva comparativa al incorporar a varios países, entregando lineamientos relacionados con las actitudes, percepciones y participación de los jóvenes en las diferentes instancias cívicas y ciudadanas (Agencia Calidad de la Educación, 2015 , 2016). Estos trabajos han aportado al conocimiento y comprensión de la temática, entregando elementos fundamentales que contribuyen a la mejora de la enseñanza de la ciudadanía en el sistema educativo.

4 ICCS sigla en inglés de International Civic and Citizenship Education Study. 
En el ámbito de la educación media técnico profesional (EMTP), varios estudios se centran en las características de sus estudiantes y las trayectorias de sus egresados (Larrañaga, Cabezas y Dussaillant, 2014). Ellos abordan las diferencias en términos de rendimiento académico entre esta modalidad y la enseñanza científico humanista (Farías y Carrasco, 2012), así como también las aspiraciones de los jóvenes egresados y la articulación de la EMTP con la educación superior, aportando elementos relevantes para su análisis y comprensión (Sepúlveda y Valdebenito, 2014; Sevilla, Farías y Weintraub, 2014). Sin embargo, ningún estudio dispuso de información relacionada con las competencias para la ciudadanía en este tipo de enseñanza, por lo que este trabajo pretende aportar en el vacío existente en este aspecto.

\section{La enseñanza media técnico profesional en Chile}

En Chile, la enseñanza media tiene una duración de cuatro años y comprende las edades entre los 14 y 18 años. Existe la enseñanza media científico-humanista (EMCH) y la formación diferenciada artística y técnico profesional ${ }^{5}$, la que es ofrecida en los dos últimos años de la enseñanza media, ya que durante los dos primeros años la formación tiene un carácter general. Los Objetivos Fundamentales y Contenidos Mínimos Obligatorios para la EMTP plantean que su función básica es:

ofrecer a los alumnos y alumnas oportunidades de realizar aprendizajes en un campo de especialización que facilite su acceso a un primer trabajo remunerado, atendiendo a sus intereses, aptitudes y disposiciones vocacionales, mediante una educación técnica en el ámbito de un sector del mundo productivo que los prepare en forma efectiva para el trabajo y para responder con flexibilidad a los cambios tecnológicos (Decreto 220, 1998, p. 285).

De esta manera, la EMTP se presenta como "la modalidad de educación responsable de ofrecer a los jóvenes una formación integral

Los centros educativos que imparten la EMTP se denominan: liceos técnicos de enseñanza media profesional, liceos polivalentes y politécnicos, estos últimos imparten la modalidad científico-humanista y la técnico-profesional. 
en el ámbito de una especialidad que facilite su inserción laboral, así como también la continuación de sus estudios sistemáticos" (Sevilla, 2011 , p. 8). Debido a ello, este tipo de formación ha tenido que reorganizar su oferta curricular en concordancia con los cambios tecnológicos y productivos que se han experimentado en el mercado laboral (Castro y Orellana, 2010).

La EMTP se aborda desde la perspectiva modular basada en el enfoque de competencias en el cual se proporciona la formación teórica y práctica y se integran los aspectos del saber y del saber hacer (Sepúlveda, 2009), definiendo al currículo por objetivos terminales asociados con un perfil de egreso de cada especialidad ${ }^{6}$. Este da cuenta de las competencias que debe alcanzar un egresado al finalizar dicha modalidad de enseñanza y está en concordancia con los requerimientos del mercado laboral. De esta manera, la elección de una especialidad por parte de los estudiantes, está en correspondencia, por un lado, con las demandas del sector productivo y, por otro, se encuentra determinada por los factores socioeconómicos, culturales y sociales. Estos juegan un rol importante, ya que los alumnos y alumnas de EMTP tienen mayor probabilidad de pertenecer a niveles socioeconómicos bajos (Arias, Farías, González-Velosa y Russi, 2015; Larrañaga, Cabezas y Dussaillant, 2013); de ahí que esta modalidad educativa represente una oportunidad para estos jóvenes, pues en poco tiempo les permite acceder al campo laboral con conocimientos en una especialidad específica.

\section{El currículo de la enseñanza media y la formación para la ciudadanía}

A partir del currículo escolar planteado por la reforma educativa en 1999, en Chile se han realizado esfuerzos en formación para la ciudadanía, dado que esta área fue deficitaria durante la dictadura militar que careció de cultura política democrática ${ }^{7}$. De hecho, la

\footnotetext{
Las bases curriculares del 2013 definen 34 especialidades agrupadas en 15 sectores económicos.

En 2000 se llevó a cabo una medición internacional de Educación Cívica que dejó a la vista la carencia de formación ciudadana en los estudiantes. Esta medición fue realizada por la International Association for the Evaluation of Educational Achievement (IEA).
} 
cultura política del periodo se caracterizaba por relaciones jerárquicas y autoritarias, con fuerte presencia policial, siendo los valores políticos predominantes el respeto por el orden y por la autoridad. Ambos elementos garantizaban una vida política y social sin riesgos, que desembocaba en formas de obediencia social (Monsálvez, 2012; Scheele, 2010), donde la participación de la ciudadanía en los asuntos políticos estaba limitada.

El currículo escolar de la época contempló la asignatura de Educación Cívica y la Economía como una manera de impartir conocimientos acerca de la Constitución Política, el funcionamiento del sistema político y económico y los derechos fundamentales. Es así como la cultura política que se quiso conservar y reproducir en el currículo respondía a un conjunto de valores, sentimientos, creencias, conocimientos y opiniones que un grupo de individuos tenía hacia los objetivos políticos que tiene una nación (Bosch y Orriols, 2011).

Luego de la recuperación democrática fue necesario reforzar e incorporar una serie de contenidos que les permitiera a los estudiantes conocer las características del sistema democrático y desarrollar habilidades y actitudes para desenvolverse en este escenario. Para ello fue necesario un cambio curricular que iba desde la educación cívica tradicional, minimalista y enfocada en la concepción de un ciudadano normativo hacia uno que contenía una concepción ciudadana moderna y maximalista basada en valores democráticos y universales de la ciudadanía cuya finalidad es formar para una cultura política democrática (Bascopé, Cox y Lira, 2015).

De esta manera en el año 1997, con la reforma a la educación básica se introdujeron los Objetivos Fundamentales (OF) relacionados con los aprendizajes que los alumnos y alumnas deben alcanzar al finalizar los distintos niveles educativos; los Contenidos Mínimos Obligatorios (CMO) que complementan a los OF y aluden a lo que los docentes deben enseñar; y los Objetivos Fundamentales Transversales (OFT) los cuales abordan los fines generales de la educación: conocimientos, habilidades, actitudes, valores y comportamientos que los estudiantes deben alcanzar y desarrollar en el ámbito personal, intelectual, moral y social. Dentro de estos últimos están también 
los diferentes aspectos de la formación ciudadana. Luego, en el año 1998, con la aprobación del marco curricular, dichos objetivos se extendieron a la enseñanza media, reafirmando con ello su carácter. De esta manera, la formación ciudadana está presente en la actualidad en toda la educación obligatoria.

En relación con lo anterior, el informe de la Comisión Ciudadana (2004) para el Ministerio de Educación de Chile (Mineduc) estableció que en la enseñanza media los OFT apelan a la responsabilidad que tienen todas las disciplinas en la formación ciudadana, ya que de esta manera se crean oportunidades de aprendizaje para que los estudiantes adquieran y desarrollen competencias para una ciudadanía participativa, crítica y responsable.

Las actualizaciones curriculares de los años 2005 y 2009 consideran aspectos por desarrollar relativos a la formación para la ciudadanía que tienen relación con:

- las actitudes personales,

- el aprendizaje y el conocimiento,

- relaciones con los demás,

- los derechos y deberes ciudadanos,

- disciplina de estudio y trabajo personal,

- el trabajo en equipo,

- el diálogo y manejo de conflictos.

Esta actualización se enfoca en la adquisición de competencias ciudadanas y sigue los lineamientos propuestos por la Organización para la Cooperación y Desarrollo Económico (OCDE) en torno a las competencias. Estos hacen referencia a la capacidad de articular y movilizar los aprendizajes, de manera que se expresen en la acción y en la utilización de conocimientos, habilidades y actitudes que los alumnos hayan aprendido, ya sea en contextos formales o informales (OCDE, 2006).

\section{Las competencias para la ciudadanía}

Para entender la función que cumplen las competencias para la ciudadanía es importante referirse al concepto de ciudadanía, el que 
está en relación con las transformaciones y requerimientos del mundo actual.

Abordando el concepto solo desde la perspectiva jurídica, la ciudadanía supone un compromiso entre el Estado y la persona, por lo que sugiere el ejercicio de sufragio, optar a cargos de elección popular y a los aspectos que la Constitución y la ley confieren. Sin embargo, la ciudadanía no está limitada a esta condición, sino que también tiene alcances relevantes relativos a los derechos de igualdad y dignidad entre las personas. En este marco, todas ellas merecen respeto (Cortina, 1997) y se debe reconocer que la integración del individuo en la comunidad política tiene que incluir a las minorías, permitiendo la expresión de las ideas de todos los grupos existentes en la sociedad con tal de favorecer la inclusión (Morín, 2001). Por lo tanto, la adhesión a la ciudadanía requiere de unos valores propios de la vida democrática, pero también necesita que las personas adquieran conocimientos y habilidades que les permitan participar en la vida social.

La ciudadanía implica conocimiento y capacidad de diálogo para la comprensión de los asuntos públicos. Esto se adquiere en la vida diaria, en las comunidades en las que las personas se desenvuelven, la familia, la escuela, las asociaciones o el barrio. Constituye una actividad social que se construye y realiza en conjunto con los miembros de la sociedad con el propósito de participar en la vida cívica, dentro de cual el individuo puede desarrollar cualidades y capacidades políticas que le permitan relacionarse con otros ciudadanos y con las instituciones del Estado de manera activa y propositiva, con determinadas competencias para responder a los requerimientos sociales y laborales.

Las competencias para la ciudadanía tienen un rol importante, ya que proporcionan herramientas elementales para desenvolverse en la sociedad. Responden a las demandas de la vida moderna, contribuyendo al bienestar personal y social (Bolívar, 2007). Mediante ellas, los sujetos intentan comprender la realidad social en la que viven, se sienten parte de la realidad en la que se desenvuelven y contribuyen a su mejora, ejerciendo la ciudadanía en una sociedad plural. 
El carácter integrador de la competencia posibilita la adaptación y la transferencia permite ejecutar tareas y enfrentar las situaciones que se presentan en los diferentes contextos en los que se desenvuelve la persona.

La educación técnico profesional no solo proporciona aprendizaje cualificado, mediante la adquisición y el desarrollo de competencias propias en una especialidad determinada, sino que también favorece la consecución de competencias básicas que son fundamentales para relacionarse en el mundo laboral y social; dado que dichas competencias van definiendo la formación de manera integral, abordando las diferentes dimensiones en las que se desarrolla el trabajo (Mertens, 2000; Zabala y Arnau, 2007) y favoreciendo la interacción y actuación en diferentes ámbitos.

Aludiendo a un enfoque de formación por competencias, Tobón (2006) -basándose en los criterios de saber ser, saber conocer y saber hacer- enmarca las competencias dentro de un proceso complejo en el que las personas ponen en acción, actuación y creación los conocimientos adquiridos para resolver problemas de la vida cotidiana y del contexto laboral, por lo que las competencias son para la vida y se aprenden en el transcurso de esta.

Desde la perspectiva profesional, estas corresponden a un conjunto de elementos integrados por conocimientos, habilidades, actitudes y saberes que toman como referencia las experiencias personales y profesionales. Estos se manifiestan mediante determinados comportamientos o conductas en el contexto del trabajo y que son puestos en juego por una persona o un equipo para llevar a cabo tareas y procesos (Miranda, 2005; Navío, 2005). Este significado holístico permite entender las competencias desde las particularidades propias de la persona hasta el contexto en el que realiza sus quehaceres cotidianos.

Las competencias para la ciudadanía, enfocadas al contexto de la EMTP, se han abordado a partir de cuatro factores adaptados desde los planteamientos de Viso (2010) y de Zabala y Arnau (2007). Cada factor corresponde a una agrupación de elementos integrados 
142 LA EDUCACIÓN TÉCNICO PROFESIONAL Y LAS COMPETENCIAS PARA LA CIUDADANÍA. EL CASO DE LAS COMUNAS DE LA PROVINCIA DE CONCEPCIÓN, CHILE - O. Carrillo - P. Jurado

por capacidades y aprendizajes competenciales que, de manera transversal, se relacionan con las competencias profesionales y se detallan a continuación:

\subsection{Factor personal}

Se refiere al desarrollo de la capacidad personal para alcanzar un compromiso cívico y ético basado en la responsabilidad social. Este factor contempla cuatro capacidades que están relacionadas con el conocimiento, los valores y la comunicación. Cada una posee sus respectivos aprendizajes competenciales que a su vez ayudan a potenciarla, aumentando la capacidad para pensar críticamente, la comprensión hacia otras culturas diferentes, la resolución no violenta de conflictos, así como también la buena voluntad para proteger el entorno.

\subsection{Factor interpersonal}

El factor está integrado por tres capacidades relacionadas con la conducta, la comunicación con los otros y los proyectos individuales y colectivos que puedan desarrollar los jóvenes. Un individuo es competente en la medida en que puede establecer un diálogo socializado, mantener relaciones de convivencia positiva con los demás, colaborando y participando en la comunidad. En este factor se agrupa todo tipo de comportamientos que una persona debe tener para ser capaz de participar de forma eficiente y constructiva en la vida social, así como también resolver conflictos cuando sea necesario.

\subsection{Factor social}

El factor social lo componen dos capacidades que están relacionadas con la participación social y la apreciación de la cultura. La persona debe ser competente para la participación activa en la sociedad en los aspectos relacionados con la comprensión de la realidad, de modo de valorar e intervenir constructiva y responsablemente en ella, fomentando la justicia, la solidaridad y la democracia. 


\subsection{Factor profesional}

Este factor abarca la capacidad de interactuar y desenvolverse en el medio. Aquí la persona debe ser competente para desenvolverse de manera adecuada en el entorno laboral, utilizando sus conocimientos y habilidades adquiridas durante su proceso de formación "responsable, flexible y rigurosamente, de manera que le permita satisfacer sus motivaciones y expectativas de desarrollo profesional y personal" (Zabala y Arnau, 2007, p. 97).

\section{La EMTP y la vulnerabilidad de sus alumnos}

Plantea Touraine (1997) que la juventud es una construcción cultural y administrativa, una imagen que la sociedad tiene de sí misma. De esta manera, es un instrumento de progreso, como un elemento marginal, por lo que los años de juventud son importantes para formar capital social y lograr la inserción e integración de la persona en la sociedad.

La vulnerabilidad refleja las amenazas a las que se deben enfrentar los jóvenes, por ejemplo, en el mercado del trabajo: muchas veces la falta de experiencia, la carencia de redes sociales o el desconocimiento de las herramientas para la búsqueda de empleo los hacen más proclives a estar desempleados, subempleados o empleados con contratos precarios. De la mano, aparece la dificultad para el acceso a vivienda, contar con espacios de relación propios o la tendencia a canalizar sus ansias de participación al margen de los límites de la institucionalidad (Programa de Naciones Unidas para el Desarrollo, PNUD, 2014; Subirats, 2004). A ello se agrega que las tasas de desempleo juvenil casi siempre son más altas que las de los adultos y también son más sensibles a los efectos de una crisis económica.

Respecto de lo anterior, se asumen tres criterios que enmarcan a los estudiantes de EMTP como un grupo con características de vulnerabilidad:

- El entorno familiar y el que habitan. El primero vinculado con las características socioeconómicas y educativas de los hogares. 
Según el estudio de educación técnico profesional este tipo de formación es la opción preferida por los jóvenes de menores recursos (Larrañaga et al., 2013). El segundo aspecto se encuentra relacionado con las zonas periféricas en las que viven los estudiantes, las que al estar alejadas de los centros educativos dificultan el acceso expedito, producto de los tiempos de traslado.

- La edad de los jóvenes (la mayor parte tiene entre 17 y 18 años). Dado que se encuentran en un periodo clave de transición entre la formación y el trabajo.

- El ámbito laboral. En estrecha relación con el punto anterior, los jóvenes en edad laboral encuentran especiales dificultades de acceso al mundo del trabajo. Esto puede generar un desajuste entre unos jóvenes cada vez más instruidos y las oportunidades reales de empleo (PNUD, 2014). Ellos son especialmente vulnerables a la marginación del mundo del trabajo debido a la falta de recursos tanto personales (experiencia, redes sociales, otros) como financieros para encontrar empleo.

En coherencia con la visión de desarrollo que tiene el país, la EMTP es necesaria y fundamental por dos razones "por su rol en la formación de competencias laborales, que es una de las bases de la productividad de la economía y por el papel que puede jugar en la movilidad social de los jóvenes pobres y vulnerables" (Larrañaga et al., 2013, p. 2).

Por lo tanto, los alumnos que asisten a la formación profesional, dadas sus características, pueden a través de esta modalidad educativa acceder a una mejora en sus condiciones de existencia, incluyendo su calidad de vida y los procesos de participación (Jurado, 2009), superando así la vulnerabilidad.

Frente a estos planteamientos, el siguiente estudio se ha focalizado en la consecución de dos objetivos, considerando el contexto estudiado:

- Analizar el nivel de logro alcanzado en relación con las competencias para la ciudadanía de los alumnos/as de EMTP de los establecimientos educativos de las comunas de la provincia de Concepción, Chile. 
- Analizar la importancia percibida de las competencias para la ciudadanía alcanzada por los alumnos/as de EMTP de los establecimientos educativos estudiados.

\section{Método}

Este estudio siguió un diseño mixto secuencial explicativo (Creswell, 2014), ya que considera que los resultados cuantitativos se explican con más detalle incorporando datos cualitativos, por lo que ambas orientaciones permiten comprender un mismo aspecto de la realidad (Bericat, 1998; Hernández, Fernández y Baptista, 2006).

Los establecimientos educativos a los que se tuvo acceso son de dependencia pública y subvencionada ${ }^{8}$ e imparten EMTP en las comunas de la provincia de Concepción y en el año 2013 atendieron a una población de 5.770 estudiantes, distribuidos en seis centros que imparten las siguientes especialidades, como se expresa en la siguiente tabla:

Tabla 1

Representación de las especialidades agrupadas por área

\begin{tabular}{lll}
\hline Centro educativo & Especialidades & Tipo de dependencia \\
\hline 1 & Atención de párvulos & Subvencionado \\
& Vestuario y confección textil & \\
\hline 2 & Administración & Público \\
& Contabilidad & \\
& Administración & Público \\
4 & Contabilidad & \\
& Electricidad & Subvencionado \\
5 & Atención de párvulos & \\
& Electricidad & Subvencionado \\
& Electrónica & \\
& Mecánica automotriz & \\
& Mecánica industrial & \\
\hline 6 & Telecomunicaciones & Subvencionado \\
& Electricidad & \\
& Electrónica & \\
& Mecánica automotriz & \\
& Telecomunicaciones &
\end{tabular}

Fuente: Elaboración propia.

8 Centros educativos de administración privada que reciben aportes del Estado. 
Se aplicó un muestreo no probabilístico intencional, siguiendo los siguientes criterios:

- Que los estudiantes se encontraran en cuarto medio de enseñanza técnico profesional, pues ya han transitado por el proceso de formación que les ha brindado las herramientas teóricas y prácticas para incorporarse al mundo laboral. De esta manera, la información que entreguen será de mayor calidad y claridad respecto de los procesos de adquisición y desarrollo de competencias.

- Que se encontraran cursando la formación en los establecimientos de las comunas de la provincia de Concepción, por la posibilidad de acceso al fenómeno de estudio.

- Que las especialidades que imparten los centros educativos estuviesen dispuestas a participar en el estudio.

Por lo anterior, la muestra quedó conformada de la manera en que se muestra en la Tabla 2:

Tabla 2

Composición de la muestra

\begin{tabular}{lccc}
\hline Especialidad & $\begin{array}{c}N^{0} \text { de alumnos/as } \\
\text { Aplicó el cuestionario }\end{array}$ & $\begin{array}{c}\mathrm{N}^{0} \text { de alumnos/as } \\
\text { entrevistados }\end{array}$ & $\begin{array}{c}\mathrm{N}^{0} \text { de profesores/as } \\
\text { entrevistados }\end{array}$ \\
\hline Administración y & 109 & 4 & 4 \\
contabilidad & 107 & 5 & 5 \\
Electricidad y electrónica & 46 & 2 & 2 \\
Telecomunicaciones & 56 & 2 & 2 \\
Mecánica automotriz & 20 & 1 & 1 \\
Mecánica industrial & 18 & 1 & 1 \\
Vestuario y confección & & 2 & 2 \\
textil & 66 & 17 & 17 \\
Atención de párvulos & 422 & & \\
Total & &
\end{tabular}

Fuente: Elaboración propia.

\section{Instrumentos utilizados para la recolección de datos}

En función de la metodología y de los objetivos, los instrumentos utilizados fueron el cuestionario de autopercepción de competencias para la ciudadanía, que fue aplicado a 422 estudiantes, y la entrevista 
semiestructurada, que se administró a un total de 34 personas: 17 a profesores y profesoras jefes de las especialidades que participaron en el estudio y 17 a estudiantes que finalizaron la EMTP y que hubiesen respondido con anterioridad el cuestionario de autopercepción de competencias para la ciudadanía.

El cuestionario presentaba dos partes. En la primera se contemplaron 16 variables dirigidas a conocer las características personales y sociofamiliares de los estudiantes, mientras que la segunda estuvo compuesta por 74 preguntas dicotómicas, agrupadas en factores: personal (11 ítems), interpersonal (12 ítems), social (18 ítems) y profesional (33 ítems) y que están enfocadas en conocer el nivel de logro de las competencias ciudadanas.

Las preguntas de la segunda parte fueron categorizadas en escala de apreciación y las respuestas se dieron en torno a los criterios de:

- 4 = siempre, la conducta o acción se presenta de manera continua (todo el tiempo).

- 3 = a menudo, la conducta o acción se manifiesta repetidamente.

- 2 = alguna vez, la conducta o acción se presenta en determinadas situaciones.

- 1 = nunca, la conducta $\mathrm{o}$ acción en ningún momento se realiza o manifiesta.

Estos cuatro criterios se consideraron para evitar los sesgos de respuesta intermedios, favoreciendo de esta manera la objetividad y la precisión (Castejón, 2006).

Cabe destacar que con anterioridad a su aplicación, el cuestionario fue sometido a un proceso de validación de contenidos por parte de 10 jueces externos en términos de univocidad, pertinencia e importancia, luego se procedió a la aplicación del instrumento en una muestra piloto de 36 jóvenes y, finalmente, se le realizó el análisis de confiabilidad cuyo resultado proporcionó un valor del coeficiente alfa de Cronbach de 0,907. El procesamiento de los datos cuantitativos se efectuó con el programa SPSS versión 17, con el cual se realizó un análisis descriptivo de los 422 cuestionarios aplicados. 
Por su parte, las entrevistas permitieron obtener las apreciaciones de los entrevistados respecto de las actitudes, hábitos, disposiciones ciudadanas como la participación y la opinión, entre otros elementos que son parte de las denominadas "competencias para la ciudadanía” y que fueron abordadas en el cuestionario. Este diálogo semiestructurado posibilitó la obtención de datos que facilitaron las interpretaciones para dar respuesta al fenómeno en estudio, dado que es más probable que los sujetos expresen sus puntos de vista en una situación de entrevista diseñada relativamente abierta (Flick, 2007).

Las entrevistas quedaron conformadas de la siguiente manera considerando los criterios de:

- Para la selección de los docentes, se estableció que estos fueran los profesores o profesoras jefes de los estudiantes de las especialidades que participaron en el estudio.

- Para los/las estudiantes, que hubiesen respondido el cuestionario en la etapa cuantitativa de este estudio, dentro de los cuales se seleccionó a los alumnos y alumnas de manera aleatoria, con consentimiento de las autoridades de los centros educativos, así como de los alumnos y alumnas escogidos.

Tabla 3

Resumen de los tutores entrevistados

\begin{tabular}{|c|c|c|c|c|c|c|}
\hline \multirow[t]{2}{*}{ Centro } & \multirow[t]{2}{*}{ Ubicación } & \multirow[t]{2}{*}{ Especialidad } & \multicolumn{3}{|c|}{ Entrevistado } & \multirow[t]{2}{*}{ Profesión } \\
\hline & & & Sexo & Edad & $\begin{array}{l}\text { Años } \\
\text { Exp. }\end{array}$ & \\
\hline 1 & Concepción & $\begin{array}{l}\text { Atención de párvulos } \\
\text { Confección textil y } \\
\text { vestuario }\end{array}$ & $\begin{array}{l}M \\
F\end{array}$ & $\begin{array}{l}63 \\
62\end{array}$ & $\begin{array}{l}37(35)^{*} \\
32(30)^{*}\end{array}$ & $\begin{array}{l}\text { Profesor de Historia y } \\
\text { Geografía } \\
\text { Profesora de Filosofía }\end{array}$ \\
\hline 2 & $\begin{array}{l}\text { Concepción } \\
\text { (Coronel) }\end{array}$ & $\begin{array}{l}\text { Administración } \\
\text { Contabilidad }\end{array}$ & $\begin{array}{l}M \\
F\end{array}$ & $\begin{array}{l}60 \\
35\end{array}$ & $\begin{array}{r}39(39)^{*} \\
5(1)^{*}\end{array}$ & $\begin{array}{l}\text { Profesor media tecnológica } \\
\text { Contador } \\
\text { Contador auditor }\end{array}$ \\
\hline 3 & $\begin{array}{l}\text { Concepción } \\
\text { (Lota) }\end{array}$ & $\begin{array}{l}\text { Administración } \\
\text { Contabilidad }\end{array}$ & $\begin{array}{l}M \\
M\end{array}$ & $\begin{array}{l}59 \\
34\end{array}$ & $\begin{array}{l}35(35)^{*} \\
10(10)^{*}\end{array}$ & $\begin{array}{l}\text { Profesor media tecnológica } \\
\text { Contador auditor }\end{array}$ \\
\hline 4 & Concepción & $\begin{array}{l}\text { Atención de párvulos } \\
\text { Electricidad }\end{array}$ & $\begin{array}{l}F \\
M\end{array}$ & $\begin{array}{l}50 \\
32\end{array}$ & $\begin{array}{r}29(4)^{*} \\
9(4)^{*}\end{array}$ & $\begin{array}{l}\text { Educadora de párvulos } \\
\text { Ingeniero eléctrico }\end{array}$ \\
\hline
\end{tabular}


5 Concepción Electricidad

(San Pedro Electrónica

de la Paz) Mecánica automotriz

Mecánica industrial

Telecomunicaciones

$\begin{array}{ll}\text { F } & 54 \\ \text { F } & 34 \\ \text { F } & 33 \\ \text { M } & 36 \\ \text { M } & 40\end{array}$

$30(20) *$ Ingeniero eléctrico

$9(9) *$ Profesora de Historia y

$6(3) *$ Geografía

$12(10) *$ Profesora de Historia y

$25(5) *$ Geografía

Profesor de Educación Física Ingeniero electrónico

6 Concepción Electricidad

Electrónica

Mecánica automotriz

Telecomunicaciones
M 47

M 64

M 60

M 59

24 (18)* Profesor de Educación Física

40 (20)* Profesor de Matemática

40 (40)* Profesor de Matemática 40 (25)* Ingeniero eléctrico

El $(*)$ indica los años de experiencia en el centro educativo.

Fuente: Elaboración propia.

Tabla 4

Resumen de los alumnos entrevistados

\begin{tabular}{|c|c|c|c|c|c|}
\hline \multirow[t]{2}{*}{ Centro } & \multirow[t]{2}{*}{ Ubicación } & \multirow[t]{2}{*}{ Especialidad } & \multicolumn{2}{|c|}{ Entrevistado } & \multirow[t]{2}{*}{ Pertenece asociación } \\
\hline & & & Sexo & Edad & \\
\hline \multirow[t]{2}{*}{1} & Concepción & Atención de párvulos & $F$ & 17 & No \\
\hline & & $\begin{array}{l}\text { Confección textily } \\
\text { vestuario }\end{array}$ & $\mathrm{F}$ & 17 & No \\
\hline \multirow[t]{2}{*}{2} & Concepción & Administración & $\mathrm{F}$ & 17 & No \\
\hline & (Coronel) & Contabilidad & M & 17 & No \\
\hline \multirow[t]{2}{*}{3} & Concepción (Lota) & Administración & $\mathrm{F}$ & 17 & Grupo juvenil iglesia \\
\hline & & Contabilidad & M & 17 & Grupo juvenil iglesia/Fútbol \\
\hline \multirow[t]{2}{*}{4} & Concepción & Atención de párvulos & $\mathrm{F}$ & 18 & Refuerzo escolar a niños \\
\hline & & Electricidad & M & 17 & Grupo juvenil iglesia \\
\hline \multirow[t]{5}{*}{5} & Concepción ISan & Electricidad & M & 17 & No \\
\hline & Pedro de la Pazl & Electrónica & M & 17 & Básquetbol (ACLE) \\
\hline & & Mecánica automotriz & $\mathrm{F}$ & 17 & No \\
\hline & & Mecánica industrial & M & 18 & Scout \\
\hline & & Telecomunicaciones & M & 17 & Artes marciales \\
\hline \multirow[t]{4}{*}{6} & Concepción & Electricidad & M & 17 & Fútbol/Básquetbol \\
\hline & & Electrónica & M & 17 & No \\
\hline & & Mecánica automotriz & M & 17 & Descenso en bicicleta \\
\hline & & Telecomunicaciones & M & 18 & \\
\hline
\end{tabular}

Fuente: Elaboración propia.

Para la interpretación de las entrevistas, estas se transcribieron, se ordenaron, se analizaron con el programa Atlas Ti, versión 5.0. Luego se establecieron las unidades de análisis que correspondían a los párrafos -pues daban información significativa- y se generaron categorías que se relacionaron con los objetivos planteados, en función de los factores determinados en el cuestionario. La codificación, que correspondió a la agrupación y relación de la información (Bruner, 
150 LA EDUCACIÓN TÉCNICO PROFESIONAL Y LAS COMPETENCIAS PARA LA CIUDADANÍA. EL CASO DE LAS COMUNAS DE LA PROVINCIA DE CONCEPCIÓN, CHILE - O. Carrillo - P. Jurado

1998) contribuyó a aglutinar los acontecimientos que compartían características semejantes y que podían ser etiquetadas en la categoría determinada.

En la siguiente tabla se muestran las categorías con sus respectivas subcategorías:

Tabla 5

Categorías y subcategorías de análisis de entrevistas

\begin{tabular}{ll}
\hline Categorías & Subcategorías \\
\hline Factor personal & - Conducta y características personales \\
Factor interpersonal & - Relaciones entre los alumnos \\
& - Temas de conversación, información e interés \\
& - Normas \\
- Pertenencia asociación \\
- Ractor social & - Relaciones entre los alumnos en las empresas e instituciones \\
Factor profesional & - Currículo \\
& - Estrategia de aprendizaje \\
& - Prácticas en las empresas e instituciones \\
\hline
\end{tabular}

Fuente: Elaboración propia.

\section{Resultados}

En relación con los objetivos planteados y al método utilizado, la presentación de los resultados se abordará en conjunto para demostrar que hay complementariedad entre los datos, lo que fortalece la comprensión de los mismos.

El análisis del cuestionario de autopercepción de competencias ciudadanas arrojó un nivel de autopercepción positiva o alta de la competencia, como se puede apreciar en la siguiente tabla: 
Tabla 6

Medias de autopercepción de competencia para la ciudadanía por factor

\begin{tabular}{ll}
\hline Factor & Media \\
\hline Personal & 3,43 \\
Interpersonal & 3,35 \\
Social & 3,23 \\
Profesional & 3,37 \\
\hline
\end{tabular}

Fuente: Elaboración propia.

Para facilitar el análisis, se procedió a la agrupación de los criterios de respuesta donde 1 y 2 constituyen una autopercepción negativa de la competencia; entre 2 y 3 no es positiva ni negativa; y para 3 a 4 el nivel alcanzado es alto o positivo.

En referencia a los factores, se exponen los siguientes resultados:

\section{a. Factor personal}

El nivel de dominio alcanzado por la autopercepción de las competencias en este factor es positivo o alto, siendo la media de 3,43. Destacan los ítems "Valoro la vida de las personas" y "Valoro la libertad", que puntúan sobre la media como se puede observar en la Tabla 7 en la que también se aprecian algunos ítems con una valoración menor como "Me adapto a la opinión de la mayoría" con 2,7. 
152 LA EDUCACIÓN TÉCNICO PROFESIONAL Y LAS COMPETENCIAS PARA LA CIUDADANÍA. EL CASO DE LAS COMUNAS DE LA PROVINCIA DE CONCEPCIÓN, CHILE - O. Carrillo - P. Jurado

Tabla 7. Medias por ítems del factor personal

\begin{tabular}{llll}
\hline Factor & Media & Ítems & Media \\
\hline & 1 & Estoy en contra de la violencia \\
& 2 & Pienso que existen cosas buenas en el mundo & 3,54 \\
& 3 & Valoro la vida de las personas & 3,32 \\
& 4 & Me gusta ayudar a las personas & 3,72 \\
& 5 & Valoro la libertad & 3,42 \\
& 6 & Todos los seres humanos somos iguales y tenemos la misma & 3,76 \\
Personal & 3,37 & importancia & \\
& 7 & Creo que con el diálogo se pueden arreglar los problemas & 3,40 \\
& 8 & Me adapto a la opinión de la mayoría & 2,72 \\
& 9 & Prefiero convencer antes que imponer lo que quiero & 3,15 \\
& 10 & Prefiero ver los aspectos positivos en vez de los negativos de & 3,13 \\
& & los demás & \\
& 11 & Puedo resolver de manera responsable las dificultades que se & 3,23 \\
& & me presentan
\end{tabular}

Fuente: Elaboración propia.

En este aspecto y en relación con la categoría conducta y características personales de la Tabla 5 se destacan respuestas como "todo bien, con mis compañeros, todo fenomenal, todos nos llevamos bien, no hay problemas con nadie" ( $5^{9}$, alumno de Telecomunicaciones), lo que va en relación con la comprensión hacia los otros y la resolución no violenta de los conflictos.

\section{b. Factor interpersonal}

El nivel de dominio alcanzado en este factor es de 3,35 por lo que la autopercepción de la competencia es alta. Destacan los ítems "En el trabajo en grupo aporto todo lo que sé", "Siempre asumo las tareas que se me asignan". Como se visualiza en la Tabla 8 uno de los ítems que tiene una puntuación bajo la media es "Cuando trabajo en equipo me agrada que me hagan preguntas", el cual se corresponde con las expresiones dadas por los alumnos entrevistados: "Soy como bien reservado para mis cosas, cuando por ejemplo piden la opinión pocas veces hablo" (4, alumno de Electricidad); lo mismo ocurre con el ítem 22 que se explica a través de las percepciones de los entrevistados "Me gusta tomar mis propias decisiones, no me gusta estar siguiendo

9 Como se ha podido observar en la Tabla 4 el número hace referencia al centro educativo al que pertenece la persona entrevistada. 
a los demás, muchas veces me desagrada, por eso también la parte del trabajo en equipo me cuesta" (6, alumno de Electricidad).

Tabla 8

Medias por ítems del factor interpersonal

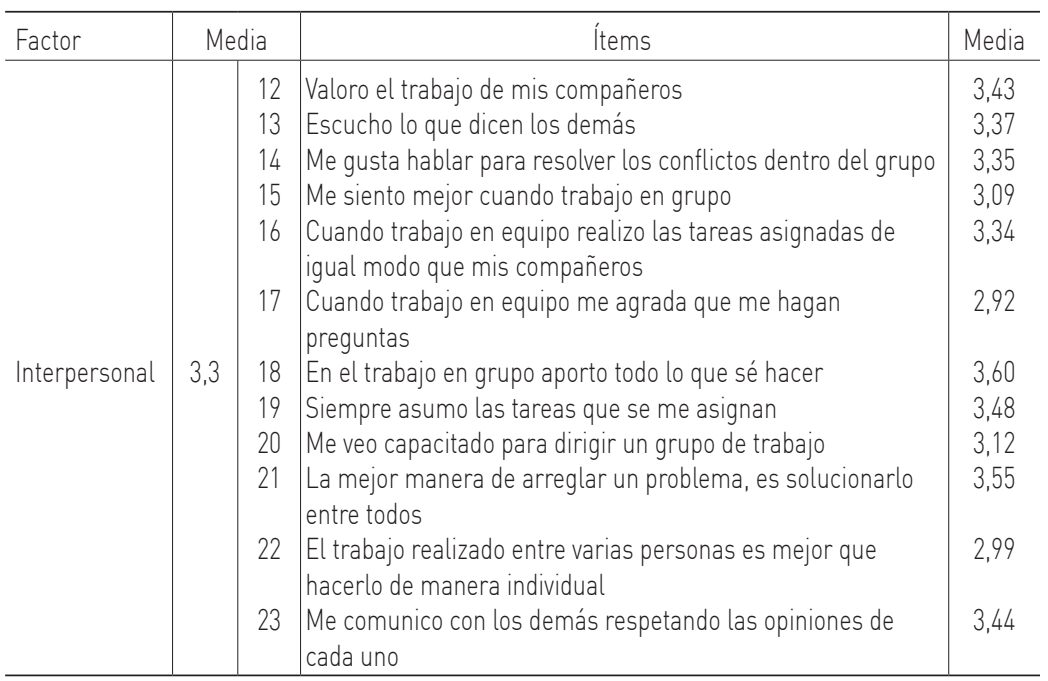

Fuente: Elaboración propia.

Este factor se centra en la comprensión hacia los demás y la comunicación mediante el diálogo y en él intervienen las subcategorías "Relaciones entre los alumnos" y "Temas de conversación". Los resultados reflejan que en los jóvenes prevalece el diálogo socializado en torno a los temas que les gustan "generalmente hablamos de nosotros, de lo que nos pasa en nuestra vida cotidiana o lo que nos gusta o planeamos hacer" (3, alumno de Administración). En el marco de la convivencia se destaca la importancia del tema de las normas, "las normas son muy necesarias" (1, alumna de Vestuario y confección textil), aunque a veces cueste acatarlas, los estudiantes expresan que son necesarias sobre todo "para cumplirlas, para mantener un orden" (5, alumno de Electricidad).

Los profesores, en coherencia con los alumnos, destacan las buenas relaciones entre pares, el respeto y el buen ambiente predominante "se llevan bien, se respetan, dentro de la palabra respeto, se manejan relativamente bien, incluso cuando uno de 
154 LA EDUCACIÓN TÉCNICO PROFESIONAL Y LAS COMPETENCIAS PARA LA CIUDADANÍA. EL CASO DE LAS COMUNAS DE LA PROVINCIA DE CONCEPCIÓN, CHILE - O. Carrillo - P. Jurado

ellos se encuentra en problemas, se apoyan" (3, profesora jefe de Contabilidad).

\section{c. Factor social}

El nivel alcanzado en este factor es de 3,72, las preguntas están dirigidas a la convivencia, a la relación armoniosa entre los integrantes de la sociedad y están en correspondencia con las subcategorías planteadas en la Tabla 5. Como se aprecia en la Tabla 9, se destacan los ítems "Apoyo a quienes me necesitan", "Todos tenemos los mismos derechos en nuestra sociedad", "Trato de actuar honestamente" y "Respeto a los demás", demostrando que los estudiantes han adquirido las competencias. "Antes todos querían hablar, ahora estamos cada uno aprendiendo competencias blandas, que uno levanta la mano, respeta y se nota el nivel" (4, alumna de Atención de párvulos). Además, los profesores jefes plantean que son chicos educables y receptivos, que reconocen cuando han cometido alguna falta de respeto hacia los demás, "en general son respetuosos, levantan la mano, piden la opinión, participan” (2, profesor jefe de Contabilidad).

Las preguntas relacionadas con la participación en la vida comunitaria como "Participo en alguna asociación" y "Participo como voluntario en tareas comunitarias" son las que tienen una autopercepción media (que no es positiva ni negativa), pero que están bajo la media del factor. En este aspecto destacan otros estudios realizados (Muñoz y Torres, 2014) que plantean que las preocupaciones del estudiantado están focalizadas en los proyectos personales, mientras que los proyectos sociales o de carácter colectivo son una opción de futuro. Pese a que ninguno de los entrevistados señaló participar en tareas voluntarias, ellos indican que desarrollan actividades que responden a sus propios intereses y que las canalizan en organizaciones religiosas como la iglesia, "yo pertenezco al coro, yo toco un instrumento, enseño a las personas que quieran aprender" (2, alumno de Contabilidad). 
Tabla 9

Medias por ítems del factor social

\begin{tabular}{|c|c|c|c|c|}
\hline Factor & Media & & Ítems & Media \\
\hline Social & 3,18 & $\begin{array}{l}24 \\
25 \\
26 \\
27 \\
28 \\
29 \\
30 \\
31 \\
32 \\
33 \\
34 \\
35 \\
36 \\
37 \\
38 \\
39 \\
40\end{array}$ & $\begin{array}{l}\text { Respeto a los demás } \\
\text { Hago amigos con facilidad } \\
\text { Ceder un poco es bueno para solucionar problemas } \\
\text { Siento que soy respetado por quienes me rodean } \\
\text { Trato de actuar honestamente } \\
\text { Tengo facilidad para relacionarme con desconocidos } \\
\text { Me gusta expresar lo que pienso sobre un tema } \\
\text { Apoyo a quienes me necesitan } \\
\text { Soy consciente de que las personas tienen limitaciones } \\
\text { Sé lo que son las elecciones democráticas } \\
\text { Participo en alguna asociación } \\
\text { Cumplo con las normas de convivencia } \\
\text { Creo que es necesario votar cuando hay elecciones } \\
\text { Acostumbro a ser amable con los demás } \\
\text { Participo como voluntario en tareas comunitarias } \\
\text { Todos tenemos los mismos derechos en nuestra sociedad } \\
\text { Espero mi turno para participar en una conversación y hablo solo } \\
\text { cuando me corresponde }\end{array}$ & $\begin{array}{l}3,56 \\
3,17 \\
3,14 \\
3,22 \\
3,59 \\
3,03 \\
3,32 \\
3,67 \\
3,52 \\
3,40 \\
2,20 \\
3,23 \\
3,08 \\
3,46 \\
2,05 \\
3,60 \\
3,16\end{array}$ \\
\hline & & 41 & Estoy al día de las noticias de actualidad & 2,91 \\
\hline
\end{tabular}

Fuente: Elaboración propia.

\section{d. Factor profesional}

En este factor la media es de 3,37, lo que indica que la autopercepción es alta o positiva. Las preguntas tienen correspondencia con la capacidad de desenvolverse utilizando conocimientos y habilidades en el mundo laboral. Como se observa en la Tabla 10 los ítems "Cuando hago un trabajo me interesa hacerlo bien", "Sé que para trabajar he de tener un contrato laboral", "Me puedo adaptar a las nuevas tecnologías que se incorporan en el mundo laboral" están sobre la media, por lo que la autopercepción de la competencia tiene un nivel alto. Sin embargo, los ítems "Tengo claras las funciones de los sindicatos" y "Sé cuándo tengo derecho a cobrar el seguro de cesantía" tienen puntuaciones bajo la media, aunque en las opiniones dadas por los estudiantes estos reconocen que tienen algunos conocimientos respecto de la normativa "no puedo decir que, al cien por ciento, pero sí tengo algunos conocimientos al respecto en el ámbito de la normativa... he leído el Código Laboral" (5, alumno de Electrónica), en tanto otros admiten que es un tema que se ha tratado en clases: "Sí, normativa laboral y previsional en una asignatura" (3, alumna de Contabilidad). 
Por su parte los profesores y profesoras coinciden en que los estudiantes sí están al tanto de las normas legales, ya que son parte de las materias que se imparten en el currículo de la formación profesional: "Tienen un ramo que se llama Derecho Laboral y Previsional y, en ese sentido, deberían saber no sé si a cabalidad, pero sí los conocen, pero no sé a qué nivel de conocimiento y de manejo de las leyes laborales" (3, profesora jefe de Contabilidad).

Coincidiendo con lo planteado en las entrevistas, los alumnos tienen una visión favorable del mundo profesional y lo expresan en la subcategoría "Prácticas en las empresas", destacando la buena disponibilidad de quienes les acogen, lo que repercute a su vez en la buena realización de las labores y en la disponibilidad para llevarlas a cabo con expectativas de mejora.

Los tutores enfatizan la relevancia de estas para el fortalecimiento de las competencias profesionales. En la subcategoría "Adaptación laboral", profesores, profesoras y estudiantes ven positivamente la adaptación, sobre todo para la realización de tareas asignadas para adquirir conocimientos en nuevas tecnologías. 
Tabla 10

\section{Medias por ítems del factor profesional}

\begin{tabular}{|c|c|c|c|c|}
\hline Factor & Media & & Ítems & Media \\
\hline Profesional & 3,34 & $\begin{array}{l}42 \\
43 \\
44 \\
45 \\
46 \\
47 \\
48 \\
49 \\
50 \\
51 \\
52 \\
53 \\
54 \\
55 \\
56 \\
57 \\
58 \\
59 \\
60 \\
61 \\
62 \\
63 \\
64 \\
65 \\
66 \\
67 \\
68 \\
69 \\
70 \\
71 \\
72 \\
73 \\
74\end{array}$ & $\begin{array}{l}\text { Tengo facilidad para realizar nuevas tareas } \\
\text { Me relaciono bien con mis compañeros de estudio o de } \\
\text { trabajo } \\
\text { Un día más en el trabajo o en el colegio es una nueva } \\
\text { oportunidad para aprender } \\
\text { Acepto que supervisen el trabajo que realizo } \\
\text { Acepto consejos para mejorar mi trabajo } \\
\text { Aguanto bien todas las horas de trabajo o de colegio } \\
\text { Estaría dispuesto a realizar otros trabajos si fuera necesario } \\
\text { Soy puntual a la hora de llegar a los sitios o al trabajo } \\
\text { Conozco las normas de prevención de riesgos laborales } \\
\text { Conozco los principales derechos y deberes del trabajador } \\
\text { Puedo hablar con mis superiores para solicitar mejores } \\
\text { condiciones de trabajo o estudio } \\
\text { Tengo claras las funciones de los sindicatos } \\
\text { Sé que para trabajar he de tener un contrato laboral } \\
\text { Entiendo bien mi horario de trabajo o de clases } \\
\text { Sé qué hacer si se vulneran mis derechos laborales } \\
\text { Sabría explicar que es una enfermedad o accidente laboral } \\
\text { Entiendo los datos de un contrato laboral } \\
\text { Sé cuándo tengo derecho a cobrar el paro lseguro de } \\
\text { cesantía) } \\
\text { Cuando hago un trabajo me interesa hacerlo bien } \\
\text { Me gusta leer cosas nuevas acerca de mi trabajo } \\
\text { Puedo relacionarme o trabajar con nuevos compañeros } \\
\text { Puedo cambiar de tarea en el trabajo cuando sea necesario } \\
\text { Sé trabajar en equipo } \\
\text { Me adapto bien a un cambio de espacio en el trabajo } \\
\text { Puedo hacer aportaciones que mejoren el trabajo } \\
\text { El trabajo bien realizado es muy valorado } \\
\text { Puedo suponer por adelantado el tiempo que necesito para } \\
\text { hacer bien un trabajo } \\
\text { Me gusta planificar mi jornada laboral } \\
\text { Me gusta ponerme a trabajar antes de que me lo tengan que } \\
\text { recordar } \\
\text { El trabajo que puedo hacer hoy no lo dejo para mañana } \\
\text { Me atrae fuertemente conseguir los objetivos planteados } \\
\text { Acepto con facilidad nuevas responsabilidades } \\
\text { Me puedo adaptar a las nuevas tecnologías que se incorporan } \\
\text { en el mundo laboral }\end{array}$ & $\begin{array}{l}3,16 \\
3,55 \\
3,46 \\
3,44 \\
3,66 \\
3,04 \\
3,30 \\
3,30 \\
3,21 \\
3,10 \\
\\
3,26 \\
2,59 \\
3,70 \\
3,74 \\
3,18 \\
3,34 \\
3,21 \\
2,93 \\
3,76 \\
3,30 \\
3,54 \\
3,36 \\
3,54 \\
3,40 \\
3,47 \\
3,66 \\
3,24 \\
3,08 \\
3,29 \\
3,00 \\
3,52 \\
3,40 \\
3,67\end{array}$ \\
\hline
\end{tabular}

Fuente: Elaboración propia. 


\section{Conclusiones}

Como se ha observado, la integración de las competencias ciudadanas en el marco del currículo en EMTP es una condición necesaria para promover procesos de adaptación de los estudiantes y futuros profesionales a la sociedad y a los entornos laborales, entendiendo que en estos últimos se dan también relaciones sociales (Gimeno, 2002; Jurado, 2009; Viso, 2010).

Los factores que hemos podido valorar (personal, interpersonal, social y profesional) en relación con el logro de las competencias para la ciudadanía por parte de los alumnos de EMTP son altos, con una media de 3,34.

El logro de las competencias se vincula directamente con el desarrollo del currículo en este tipo de formación que incluye la enseñanza de asignaturas humanistas (Filosofía, Leguaje y Comunicación, Historia y Ciencias Sociales) y que junto con las asignaturas propias de la especialidad refuerzan y fortalecen las competencias para la ciudadanía, favoreciendo la actuación ciudadana de los alumnos y alumnas. No obstante esto no necesariamente esto es reflejo de lo que sucede a diario en el contexto educativo, ya que se han encontrado opiniones contrarias entre los profesores y profesoras, quienes por un lado expresan que los y las estudiantes "no saben dialogar", "se quieren harto, pero se faltan el respeto"; y por otro exponen que "son educados" y "son respetuosos".

Desde la perspectiva de los entrevistados el nivel de logro de las competencias estaría influenciado por la conducta que posee el estudiante, la que es fundamental para el logro de las competencias. En este aspecto la conducta está relacionada con el entorno en el que se desenvuelve y que se visualiza en la sala de clases y en el centro educativo. Otro factor lo constituyen las normas, en este caso cómo las asumen los estudiantes, ya que son necesarias para resguardar un clima de aprendizaje para que prevalezcan las buenas relaciones entre quienes componen la comunidad educativa. Por último, los temas de conversación, información e interés de los alumnos los predisponen a una actitud favorable ante el conocimiento y aprendizaje. 
Asimismo, como parte del currículo escolar, se destaca que las prácticas en las empresas son importantes no solo para la transferencia de los aprendizajes, sino también para el aprendizaje en contexto, pues favorecen el ejercicio de los conocimientos y competencias adquiridas, y fomentan el desarrollo de nuevos aprendizajes y competencias para el trabajo. Otro factor importante se relaciona con las estrategias de aprendizaje utilizadas por los docentes que facilitan o condicionan la adquisición de conocimientos y de competencias por parte de los alumnos y alumnas de EMTP.

Con respecto a la importancia percibida por parte de los estudiantes hacia sus propias competencias para la ciudadanía, se concluye que estas son vistas como positivas y coinciden con los resultados del cuestionario, lo que ha quedado demostrado en las entrevistas y evidenciado en las categorías y subcategorías analizadas.

Además es importante mencionar el clima escolar, ya que como expresan algunos estudios (Fernández, 2004; Lamoyi, 2009), este influye en el logro de los objetivos, de los aprendizajes y en la adquisición de las competencias. En el caso de las competencias para la ciudadanía, fortalece la integración e identidad entre los miembros de la comunidad educativa, favorece el debate abierto en el aula, la confianza y la participación, todos ellos aspectos relevantes para el desarrollo de la ciudadanía activa, como lo han dejado en evidencia las opiniones de los entrevistados que se sintetizan en frases como: "Es agradable el ambiente que se vive acá". Ello se corresponde con las investigaciones realizadas por el Ministerio de Educación de Chile (Mineduc, 2004) en las que se concluye que el currículo da los lineamientos para hacer del centro educativo un espacio de formación ciudadana.

Finalmente, como limitación y perspectiva, hay que apuntar el interés de ampliar el estudio, ya que, por ejemplo, sería enriquecedor poder contar con las opiniones de los directivos de los centros educativos, los encargados de la EMTP y los agentes del sistema productivo que acogen a los estudiantes en las prácticas, ya que podrían aportar elementos necesarios para contrastar o verificar la información entregada por los estudiantes, pues su visión hubiese 
enriquecido el conocimiento que se tiene de las prácticas en las empresas.

\section{Referencias}

Agencia Calidad de la Educación (2015). Estudio internacional de educación cívica y formación ciudadana. Marco de evaluación y ejemplos de preguntas. Recuperado de: http://archivos.agenciaeducacion.cl/Estudio_Marco_ Evaluacion_ICCS.pdf

Agencia Calidad de la Educación (2016). Formación ciudadana en el sistema escolar chileno: una mirada a las prácticas actuales y recomendaciones de mejora. Recuperado de: http://www.agenciaeducacion.cl/wp-content/ uploads/2016/02/Estudio_Formacion_ciudadana_en_sistema_escolar_ chileno.pdf

Arias, E., Farías, M., González-Velosa, C., y Rucci, G. (2015). Educación técnico profesional en Chile. Recuperado de: https://publications.iadb. org/bitstream/handle/11319/6881/Educacion_tecnico_profesional_ Chile.PDF

Bascopé, M., Cox, C., y Lira, R. (2015). Tipos de ciudadano en los currículos del autoritarismo y la democracia. En C. Cox y J. Castillo (Eds.), Aprendizaje de la ciudadanía. Contextos, experiencias y resultados (pp. 245-482). Santiago de Chile: Ediciones Universidad Católica.

Banks, J., McGee, Ch., Cortés, C., Hann, C., Merryfield, M., Moodley, K., ... Parker, W. (2005). Democracy and diversity. Principles and concepts for educating citizens in a global age. Center for multicultural education, College of Education, University of Washington, Seatle.

Banks, J. (2008). Diversity and citizenship education in global times. En J. Arthur, I. Davies, \& C. Hahn (Eds.), The Sage handbook of education for citizenship and democracy (pp. 57-70). London: Sage Publications.

Bericat, E. (1998). La integración de los métodos cuantitativo y cualitativo en la investigación social. Barcelona: Ariel.

Bonhome, M., Cox, C., Tham, M., y Lira, R. (2015). La educación ciudadana escolar en Chile 'en acto': prácticas docentes y expectativas de participación política de estudiantes. En C. Cox y J. Castillo (Eds.), Aprendizaje de la ciudadanía. Contextos, experiencias y resultados (pp. 373-428). Santiago de Chile: Ediciones Universidad Católica.

Bolívar, A. (2007). Educación para la ciudadanía. Algo más que una asignatura. Barcelona: Graó. 
Bosch, A. y Orriols, Ll. (2011). Ciéncia politica per a principiants. Barcelona: Editorial UOC.

Bruner, J. (1988). Desarrollo cognitivo y educación. Madrid: Morata S.A.

Castro, E. y Orellana, W. (2010). La educación media técnico profesional en Chile: entre la desarticulación y la indefinición. Revista Intersecciones Educativas, 2, 73-81. Recuperado de: http://educacion.ulagos.cl/ revista/index.php/edicion-ii/item/8-edicion2-articulo-6

Cortina, A. (1997). Ciudadanos del mundo. Hacia una teoría de la ciudadanía. Madrid: Alianza Editorial.

Creswell, J. (2014). Research design: Qualitative, quantitative, and mixed methods approaches. Thousand Oaks, CA: Sage.

Decreto n 220, Ministerio de Educación. Diario Oficial de la República de Chile, 18 de mayo de 1998.

Farías, M. y Carrasco, R. (2012). Diferencias en resultados académicos entre educación técnico profesional y humanista científica en Chile. Calidad en la educación, 36, 87-121.

Fernández, T. (2004). Clima organizacional en las escuelas: un enfoque comparativo para México y Uruguay. REICE Revista Electrónica Iberoamericana sobre calidad, eficacia y cambio en educación, 2(2), 43-68. Recuperado de: www.ice.deusto.es/RINACE/reice/vol2n2/Tabare.pdf

Flick, U. (2007). Introducción a la investigación cualitativa. Madrid: Morata.

Gimeno, J. (2002). Educar y convivir en la cultura global: las exigencias de la ciudadanía. Madrid: Morata.

Hernández, R., Fernández, C., y Baptista, P. (2006). Metodología de la investigación. México: McGraw-Hill.

Jurado, P. (2009). Calidad de vida y procesos educativos (Documento interno) Universidad Autónoma de Barcelona, Barcelona, España.

Larrañaga, O., Cabezas, G., y Dussaillant, F. (2013). Estudio de la educación técnico profesional. Programa de las Naciones Unidas para el Desarrollo - Chile. Área de Reducción de la Pobreza y la Desigualdad. Recuperado de: http://www.undp.org/content/dam/chile/docs/ pobreza/undp_cl_pobreza_etp_2013.pdf

Larrañaga, O., Cabezas, G., y Dussaillant, F. (2014). Trayectorias educacionales e inserción laboral en la enseñanza media técnico profesional. Estudios Públicos, 134, 7-58. Recuperado de: https://www.cepchile.cl/cep/ site/artic/20160304/asocfile/20160304100655/rev134_OLarranagaGCabezas-FDussaillant.pdf 
Lamoyi, C. (2009). Clima organizacional: creencias compartidas, sentido de comunidad y liderazgo directivo, en escuelas secundarias de Tabasco. Recuperado de: www.eumed.net/cursecon/ecolat/mx/2009/cllb.htm

Ley no 18.962, establece la Ley Orgánica Constitucional de Enseñanza del Ministerio de Educación. Diario Oficial de la República de Chile, Santiago, Chile, 10 de marzo de 1990.

Ley n 20.370 establece la Ley General de Educación del Ministerio de Educación. Diario Oficial de la República de Chile, Santiago, Chile, 12 de septiembre de 2009.

Magendzo, A. (2003). Formación ciudadana y objetivos fundamentales transversales. Recuperado de: http://www.bcn.cl/carpeta_temas/temas_ portada.2005-10-1025.4785762907/folder.2005100formacion\%20 ciudadana\%20y\%20obj\%20transversales.pdf

Magendzo, A. y Toledo, M. I. (2009). Educación en Derechos Humanos: currículum Historia y Ciencias Sociales del $2^{\circ}$ año de enseñanza media. Estudios Pedagógicos, 1(35), 139-154. https://doi.org/10.4067/s0718-07052009000100008

Maiztegui, C. (2007). La participación como una opción transformadora en los procesos de educación ciudadana. REICE, Revista electrónica iberoamericana sobre calidad, eficacia y cambio en educación, 5(4) 144-160.

Mertens, L. (2000). La gestión por competencia laboral en la empresa y la formación profesional. Madrid: OEI.

Ministerio de Educación, Mineduc (2004). Formación ciudadana. Actividades de apoyo para el profesor. Historia y Ciencias Sociales. $1^{\circ}$ básico a $4^{\circ}$ medio. Recuperado de: www.oei.es/valores2/formacion_ciudadana.pdf

Ministerio de Educación, Mineduc (2009). Contenidos mínimos obligatorios de la educación básica y media. Recuperado de: http://www.ibe.unesco.org/ curricula/chile/cl_al_fw_2009_spa.pdf

Monsálvez, D. (2012). La dictadura militar de Augusto Pinochet como Nueva Historia Política: perspectiva historiográfica y algunos temas para su indagación. Revista Austral Ciencias Sociales, 23, 61-82.

Morín, E. (2001). Los siete saberes necesarios para la educación del futuro. Barcelona: Paidós.

Muñoz, C., Vásquez, N. y Reyes, L. (2010). Percepción del estudiantado de enseñanza básica sobre el rol del Estado, las instituciones públicas, la democracia, la ciudadanía y los derechos de las mujeres y de los inmigrantes. Estudios Pedagógicos XXXVI, 2, 153-175. 
Muñoz, C. y Torres, B. (2014). La formación ciudadana en la escuela: problemas y desafíos. Revista Electrónica Educare, 2(18), 233-245.

Navío, A. (2005). Propuestas conceptuales en torno a la competencia profesional. Recuperado de: http://www.revistaeducacion.mec.es/re337_11.htm

Organización de Cooperación y Desarrollo Económicos, OCDE (2006). La definición y selección de competencias clave. Recuperado de: www.deseco. admin.ch/.../2005.dscexecutivesummary.sp.pdf

Pagès, J. y Santisteban, A. (2009). Una propuesta conceptual para la investigación en educación para la ciudadanía. Revista Educación y Pedagogía, 21(53), 15-31.

Peña, C. (2015). Escuela y vida cívica. En C. Cox y J. Castillo (Eds.), Aprendizaje de la ciudadanía. Contextos, experiencias y resultados (pp. 25-50). Santiago de Chile: Ediciones Universidad Católica.

Programa de Naciones Unidas para el Desarrollo, PNUD. (2014). Informe sobre desarrollo humano 2014. Sostener el progreso humano: reducir vulnerabilidades y construir resiliencia. Recuperado de: http://hdr.undp. org/es/content/informe-sobre-desarrollo-humano-2014

Reyes, L., Campos, J., Osandón L., y Muñoz, C. (2013). El profesorado y su rol en la formación de los nuevos ciudadanos: desfases entre las comprensiones, las actuaciones y las expectativas. Estudios Pedagógicos, 1(39), 217-237.

Scheele, J. (2010). Entre orden y caos: la formación de la cultura política chilena desde una perspectiva histórica. Revista Pléyade 5, 2-45. Recuperado de: http://www.academia.edu/2592957/Entre_orden_y_ caos_La_formaci\%C3\%B3n_de_la_cultura_pol\%C3\%ADtica_chilena_ desde_una_perspectiva_hist\%C3\%B3rica

Sepúlveda, L. (2009). Estado y perspectivas de la enseñanza media técnico profesional en Chile: un estudio sobre las orientaciones estratégicas predominantes en los actores. Recuperado de: http://biblioteca. uahurtado.cl/ujah/reduc/pdf/pdf/txt828.pdf

Sepúlveda, L. y Valdebenito, M. (2014). Aspiraciones y proyectos de futuros de estudiantes de enseñanza técnico-profesional: ¿Es pertinente un sistema diferenciado en la enseñanza media? Polis, Revista latinoamericana, 38(13), 597-620.

Sevilla, M. (2011). Educación técnica profesional en Chile. Antecedentes y claves de diagnóstico. Recuperado de: http://www.mineduc.cl/usuarios/ mineduc/doc/201204241130130.DiagnOsticoEducaciOnTPCentrod eEstudiosMINEDUC.pdf 
Sevilla, M., Farías, M., y Weintraub, M. (2014). Articulación de la educación técnico profesional: una contribución para su comprensión y consideración desde la política pública. Calidad en la Educación, 41, 83-117. https://doi.org/10.4067/s0718-45652014000200004

Subirats, J. (Dir.) (2004). Pobreza y exclusión social. Un análisis de la realidad española y europea. Recuperado de: http://www.didacqueralt.com/ pobreza-sp.pdf

Tyson, C. \& Choon, S. (2008). Civic education, social justice and critical race theory. En J. Arthur, I. Davies, \& C. Hahn (Eds.), The Sage handbook of education for citizenship and democracy (pp. 29-39). London: Sage Publications.

Tobón, S. (2006). Formación basada en competencias: pensamiento complejo, diseño curricular y didáctica. Recuperado de: http://es.scribd.com/ doc/28137201/Formacion-Basada-en-Competencias

Touraine, A. (1997). Juventud y democracia en Chile. Recuperado de: http:// www.plataformademocratica.org/Publicacoes/7401_Cached.pdf

Viso, J. (2010). ¿Qué son las competencias? Vol. I. Madrid: Editorial EOS.

Zabala, A. y Arnau, L. (2007). 11 Ideas. ¿Cómo aprender y enseñar competencias? Barcelona: Graó.

Recibido: 31/08/2016

Aceptado: 20/04/2017 